\title{
POLAR CLASSES AND SEGRE CLASSES ON SINGULAR PROJECTIVE VARIETIES
}

\author{
SHOJI YOKURA
}

\begin{abstract}
We investigate the relation between polar classes of complex varieties and the Segre class of K. Johnson [Jo]. Results are obtained for hypersurfaces of projective spaces and for certain varieties with isolated singularities.
\end{abstract}

0. Introduction. The relation between polar classes and Chern classes (ChernMather and Chern-MacPherson classes) of singular complex varieties has been studied by several authors (Dubson, Lê, Teissier, Piene, etc.). This paper is motivated by trying to understand the relation between Chern classes and Segre classes [Jo] of singular varieties, which has not been clarified yet. As one of the steps for this we have tried to capture the relation between Segre classes $S_{*}(X)$ defined by $\mathrm{K}$. Johnson [Jo] and our Segre-Mather classes $S_{*}^{M}(X)$ defined in a similar manner to that of Chern-Mather classes $C_{*}^{M}(X)$.

Our first main theorem is

THEOREM A. Let $X^{n} \subseteq \mathbf{P}^{n+1}$ be a reduced hypersurface with $S$ denoting the singular subvariety of $X$. Then we have

$$
S_{i}(X)=S_{i}^{M}(X)+S^{i-1}\left(\mathbf{P}^{n+1}\right) \cap\left(\sum_{j} e_{j}\left[S_{n-1, j}\right]\right),
$$

where $S_{n-1, j}$ are irreducible components of dimension $n-1$ of singular subvariety $S$ of $X, e_{j}$ is the multiplicity of the Jacobian ideal in the local ring of $X$ at the generic point of $S_{n-1, j}($ e.g., see $[\mathbf{F 1}, \S 4.3])$, and $S^{i-1}\left(\mathbf{P}^{n+1}\right)$ is the $(i-1)$ st usual Segre class of $\mathbf{P}^{n+1}$.

Our second main theorem is for $X^{n} \subseteq \mathbf{P}^{2 n}$ with isolated singularities. Let $x$ be a singular point of $X$, let $P$ be a generic point off $X$, and let $H$ be a hyperplane not containing $x$. Consider the affine variety $X^{a}=X-H \subseteq \mathbf{P}^{2 n}-H=\mathbf{C}^{2 n}$. Let $\varepsilon$ be a sufficiently small positive number and let $t$ be a complex number such that $|t| \ll \varepsilon$. Shift $X^{a}$ towards the point $P$ by the length $t$. Let $S_{P, t}\left(X^{a}\right)$ denote such a shifted $X^{a}$. Then count the intersection points of $X^{a}$ and the shifted $S_{P, t}\left(X^{a}\right)$ within the $\varepsilon$-ball $B(x)$ around the singular point $x$. (For a generic point $P, S_{P, t}\left(X^{a}\right)$ and

Received by the editors October 23, 1984 and, in revised form, October 11, 1985.

1980 Mathematics Subject Classification. Primary 14C17, 14F45, 32B30; Secondary 14E25, 14 F05.

Key words and phrases. Chern-MacPherson class, Chern-Mather class, Johnson's Segre class, polar class, Todd formula, Nash blowup. 
$X^{a}$ are transverse to each other within $B_{\varepsilon}(x)$ for sufficiently small $\varepsilon$ and $t$.) This number is denoted $n(x, P, t, \varepsilon)$ and called the shift multiplicity of $x$. With this new multiplicity, our second main theorem is

THEOREM B. Let $X^{n} \subseteq \mathbf{P}^{2 n}$ be a reduced singular variety with isolated singularities $x_{1}, x_{2}, \ldots, x_{r}$. Then we have

$$
S_{i}(X)=S_{i}^{M}(X) \text { for } i<n,
$$

and

$$
S_{n}(X)=S_{n}^{M}(X)+\sum_{i=1}^{r} n_{i}\left[x_{i}\right]
$$

where $n_{i}$ is the shift multiplicity of each singularity $x_{i}$.

In $\$ 1$ we discuss Chern-Mather and Segre-Mather classes and polar classes corresponding to them. $\S 2$ is a quick review of K. Johnson's thesis [Jo]. $\$ 3$ deals with the decomposition of the class $[P(X)]$, which appears in the definition of Johnson's Segre classes $S_{*}(X)$. $\S \S 4$ and 5 contain our main results, for hypersurfaces and for $X^{n} \subseteq P^{2 n}$ with isolated singularities, respectively.

ACKNOWLEDGMENTS. I wish to thank my advisor, C. McCrory, for his guidance and encouragement, and also G. Kennedy, T. Shifrin, R. Smith, and especially R. Varley, for their valuable suggestions and comments. Also I would like to thank W. Fulton and R. MacPherson for their encouragements.

1. Chern-Mather, Segre-Mather, and polar classes. Let $X^{n}$ be a projective variety of pure dimension $n$ in the complex projective space $\mathbf{P}^{N}$. A variety is understood to be a reduced scheme (possibly reducible). Let $\operatorname{Gr}\left(T \mathbf{P}^{N}, n\right)$ be the Grassmannian bundle over $\mathbf{P}^{N}$; its fiber over a point $x \in \mathbf{P}^{N}$ is the Grassmannian $\operatorname{Gr}\left(T_{X} \mathbf{P}^{N}, n\right)$ of $n$-planes in the tangent space $T_{x} \mathbf{P}^{N}$ at $x$. Let $X_{s m}$ denote the open dense subvariety of nonsingular points. We consider the canonical embedding

$$
g: X_{s m} \hookrightarrow \operatorname{Gr}\left(T \mathbf{P}^{N}, n\right), \quad x \mapsto T_{x} X_{s m} .
$$

The closure of the image $g\left(X_{s m}\right)$ is called the Nash blowup of $X$ and is denoted by $\hat{X}$; the Nash blowup map $\nu: \hat{X} \rightarrow X$ is the restriction of the projection map $\pi$ : $\operatorname{Gr}\left(T \mathbf{P}^{N}, n\right) \rightarrow \mathbf{P}^{N}$. It is well known (e.g., [Du or Go]) that (i) $\hat{X}$ is algebraic, (ii) $\nu$ : $\hat{X} \rightarrow X$ is algebraic proper, and (iii) over $X_{s m} \nu$ is an (algebraic) isomorphism. The restriction to $\hat{X}$ of the tautological bundle on $\operatorname{Gr}\left(T \mathbf{P}^{N}, n\right)$ is called the Nash tangent bundle of $\hat{X}$ and is denoted by $\widehat{T X}$.

Definition. The ith Chern-Mather class $C_{i}^{M}(X)$ is defined by

$$
C_{i}^{M}(X)=\nu_{*}\left(c_{i}(\widehat{T X}) \cap[\hat{X}]\right),
$$

where $c_{i}(\widehat{T X})$ is the $i$ th Chern class of $\overparen{T X}$. The $i$ th Segre-Mather class $S_{i}^{M}(X)$ is defined by

$$
S_{i}^{M}(X)=\nu_{*}\left(s_{i}(\widehat{T X}) \cap[\hat{X}]\right),
$$

where $s_{i}(\overparen{T X})$ is the $i$ th inverse Chern class of $\overparen{T X}$. 
Let $\mathbf{P}(\widehat{T X})$ be the projectivization of the Nash tangent bundle, $\mathcal{O}_{\mathbf{P}(\widehat{T X})}(1)$ the dual of the tautotlogical line bundle over $\mathbf{P}(\widehat{T X}), t: \mathbf{P}(\widehat{T X}) \rightarrow \hat{X}$ the projection map, $p_{1}=\nu \cdot t$, and $\theta=c_{1}\left(\mathcal{O}_{\mathbf{P}(\widehat{T X})}(1)\right)$. Then it is well known (e.g., [K1]) that $S_{i}^{M}(X)=$ $p_{1^{*}}\left(\theta^{n-1+i} \cap[\mathbf{P}(\widehat{T X})]\right)$.

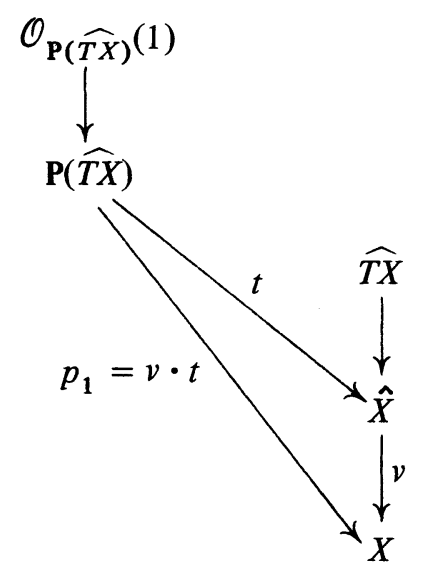

Let $\operatorname{Gr}(N, n)$ denote the Grassmannian of $\mathbf{P}^{n}$ 's in $\mathbf{P}^{N}$. We define the projective Gauss map $\hat{\gamma}: \hat{X} \rightarrow \operatorname{Gr}(N, n)$ by $\xi \mapsto \bar{\xi}$, where $\bar{\xi}$ is the unique linear subspace of $\mathbf{P}^{N}$ of dimension $n$ whose tangent space at $x$ is $\xi$. Let $\tilde{E}^{n+1}$ be the tautological bundle of rank $n+1$ over $\operatorname{Gr}(N, n)$. Let $\widetilde{T X}$ denote the pull-back of $\tilde{E}^{n+1}$ to $\hat{X}$ via $\hat{\gamma}$. Define $\gamma: \operatorname{Gr}\left(T \mathbf{P}^{N}, n\right) \rightarrow \operatorname{Gr}(N, n)$ by $\gamma(\xi)=\bar{\xi}$ just as above, so that $\hat{\gamma}$ is $\gamma$ composed with inclusion. Let $L_{\hat{X}}$ be the pull-back (via $\nu: \hat{X} \rightarrow X$ ) of the line bundle $\mathcal{O}_{X}(-1)$ on the projective variety $X$. There is an exact sequence (the Euler sequence)

$$
0 \rightarrow \pi^{*} \mathcal{O}_{\mathbf{P}^{N}}(-1) \rightarrow \gamma^{*} \tilde{E}^{n+1} \rightarrow E^{n} \otimes \pi^{*} \mathcal{O}_{\mathbf{P}^{N}}(-1) \rightarrow 0
$$

See, for instance, [GH, p. 409]. Pulling back this exact sequence via the inclusion map $i: \hat{X} \rightarrow \operatorname{Gr}\left(T \mathbf{P}^{N}, n\right)$ yields the exact sequence (cf. [GH, Sh])

$$
0 \rightarrow L_{\hat{X}} \rightarrow \widetilde{T X} \rightarrow \widetilde{T X} \otimes L_{\hat{X}} \rightarrow 0 .
$$

The Chern and Segre classes of $\widetilde{T X}$ are closely related to "polar loci". Let $A=A^{N-n+k-2}$ be a liner subspace of dimension $(N-n+k-2)$ of $\mathbf{P}^{N}$. The polar locus $P(A)$ of $X$ with respect to $A$ is defined to be the closure of the locus of points $x$ of $X_{s m}$ such that the projective tangent space $\overline{T_{x} X_{s m}}$ intersects $A$ in a space of at least $(k-1)$ dimension. Piene [Pi1, Pi2] showed that for a generic $A$, the polar locus is of codimension $k$, and that the homology class $P_{k}(X)$ represented by the polar locus is independent of the choice of a generic $A$. Explicitly, if $\operatorname{Sch}_{k}(A)$ denote the Schubert variety

$$
\{P \in \operatorname{Gr}(N, n): \operatorname{dim}(P \cap A) \geqslant k-1\},
$$

then for a generic $A$,

$$
P(A)=\nu\left(\hat{\gamma}^{-1}\left(\operatorname{Sch}_{k}(A)\right)\right), \quad \text { and } \quad P_{k}(X)=\nu_{*}\left(\left[\hat{\gamma}^{-1}\left(\operatorname{Sch}_{k}(A)\right)\right]\right) .
$$


Recall the Gauss-Bonnet Theorem (cf. [GH or KL]): The Poincaré dual of the Schubert cycle $\left[\operatorname{Sch}_{k}(A)\right]$ is equal to $(-1)^{k} c_{k}(\tilde{E})$, i.e.,

$$
\left[\operatorname{Sch}_{k}(A)\right]=(-1)^{k} c_{k}(\tilde{E}) \cap[\operatorname{Gr}(N, n)] .
$$

Thus for a generic $A$,

$$
P_{k}(X)=(-1)^{k} \nu_{*}\left(c_{k}(\widetilde{T X}) \cap[\hat{X}]\right)
$$

From the Euler exact sequence we get

$$
c_{k}(\widehat{T X})=\sum_{i=0}^{k}\left(\begin{array}{c}
n+1-i \\
k-i
\end{array}\right) c_{i}(\widetilde{T X}) \cup c_{1}\left(L_{X}^{*}\right)^{k-i}
$$

and

$$
c_{k}(\widetilde{T X})=\sum_{i=0}^{k}(-1)^{k-1}\left(\begin{array}{c}
n+1-i \\
k-i
\end{array}\right) c_{i}(\widehat{T X}) \cup c_{1}\left(L_{X}^{*}\right)^{k-i}
$$

These formulas imply the following Todd formulas for polar classes and ChernMather classes [Pi2]:

$$
C_{k}^{M}(X)=\sum_{i=0}^{k}(-1)^{i}\left(\begin{array}{c}
n+1-i \\
k-i
\end{array}\right) U^{k-i} \cap P_{i}(X),
$$

and

$$
P_{k}(X)=\sum_{i=0}^{k}(-1)^{i}\left(\begin{array}{c}
n+1-i \\
k-i
\end{array}\right) U^{k-i} \cap C_{i}^{M}(X),
$$

where $U=c_{1}\left(\mathcal{O}_{X}(1)\right)$.

Now let $A=A^{N-m-1}(m \geqslant n)$ be a linear subspace of dimension $(N-m-1)$ of $\mathbf{P}^{N}$. The polar locus $\bar{P}(A)$ of $X$ with respect to $A$ is defined to be the closure of the locus of points $x$ of $X_{s m}$ such that the projective tangent space $\overline{T_{x} X_{s m}}$ intersects $A$. In [Jo and Pil] it is shown that for a generic $A, \bar{P}(A)$ has the "expected" codimension $k=m-n+1$ and that its homology class $\bar{P}_{k}(X)$ is independent of $A$. We call this class the polar class.

Explicitly, if $\operatorname{Sch}_{1}^{\prime}(A)$ denotes the special Schubert variety

$$
\{P \in \operatorname{Gr}(N, n): P \cap A \neq \varnothing\},
$$

then for a generic $A$,

$$
\bar{P}(A)=\nu\left(\hat{\gamma}^{-1}\left(\operatorname{Sch}_{1}^{\prime}(A)\right)\right) \quad \text { and } \quad \bar{P}_{k}(X)=\nu_{*}\left(\left[\hat{\gamma}^{-1}\left(\operatorname{Sch}_{1}^{\prime}(A)\right)\right]\right) .
$$

By the Gauss-Bonnet Theorem, the Poincaré dual of the homology class [ $\left.\operatorname{Sch}_{1}^{\prime}(A)\right]$ is equal to the $(m-n+1)$ st Chern class $c_{m-n+1}(Q)$ of the tautological rank $(N-n)$ quotient bundle $Q$ of the trivial bundle $\mathscr{E}^{N+1}$ by $\tilde{E}^{n+1}$ :

$$
0 \rightarrow \tilde{E}^{n+1} \rightarrow \mathscr{E}^{N+1} \rightarrow Q^{N-n} \rightarrow 0
$$

Thus

$$
\bar{P}_{k}(X)=\nu_{*}\left(c_{k}\left(\hat{\gamma}^{*} Q\right) \cap[\hat{X}]\right)
$$


From the previous exact sequence we get the exact sequence over $\hat{X}$

$$
0 \rightarrow \widetilde{T X} \rightarrow \hat{\gamma}^{*} \mathscr{E}^{N+1} \rightarrow \hat{\gamma}^{*} Q^{N-n} \rightarrow 0
$$

Hence by the Whitney product formula

$$
\bar{P}_{k}(X)=\nu_{*}\left(s_{k}(\widetilde{T X}) \cap[\hat{X}]\right)
$$

The Euler exact sequence again yields Todd formulas, this time involving Segre classes:

$$
\bar{P}_{k}(X)=\sum_{i=0}^{k}\left(\begin{array}{c}
n+k \\
i
\end{array}\right) U^{i} \cap S_{k-i}^{M}(X)
$$

and

$$
S_{k}^{M}(X)=\sum_{i=0}^{k}(-1)^{i}\left(\begin{array}{c}
n+k \\
i
\end{array}\right) U^{i} \cap \bar{P}_{k-i}(X) .
$$

2. A quick review of $\mathbf{K}$. W. Johnson's thesis [Jo]. Johnson's Segre class $S_{*}(X)$ is defined as the relative Segre class $S(d(X), X \times X)$ of $X \times X$ with respect to the diagonal $d(X)$. We recall the details here. Let $\overline{X \times X} \stackrel{\pi}{\rightarrow} X \times X$ be the blowup of $X \times X$ along the diagonal, which is defined by some ideal sheaf $I$. Let $P(X)$ be the exceptional divisor of this blowup; i.e.,

$$
P(X)=\operatorname{Proj}\left(\bigoplus_{j \geqslant 0} I^{j} / I^{j+1}\right),
$$

which is the projectivization of the normal cone [F3]

$$
C_{d(X)}(X \times X)=\operatorname{Spec}\left(\bigoplus_{j \geqslant 0} I^{j} / I^{j+1}\right) .
$$

Note that if $X$ is of equidimension $n$, then $P(X)$ is of equidimension $2 n-1$. If we restrict $\pi$ to the exceptional divisor $P(X)$ and identify $d(X)$ with $X$, we have the projection map $p: P(X) \rightarrow X$. Let $\xi=c_{1}\left(\mathcal{O}_{P(X)}(1)\right)$. Then the $i$ th Segre(-Johnson) class $S_{i}(X)$ is defined by

$$
S_{i}(X)=p_{*}\left(\xi^{n-1+i} \cap[P(X)]\right) \in H_{2(n-i)}(X) .
$$

(For the general notion of relative Segre classes, see [F3, FL, FM1].)

Johnson studied the scheme-theoretical fiber over $x$,

$$
\operatorname{Spec}\left(\oplus I^{j} / I^{j+1}\right) \times_{X} \operatorname{Spec}(k(x)),
$$

(where $k(x)$ is the residue field $\mathcal{O}_{X, x} / M_{x}$ ) and called it the tangent star to $X$ at $x$. We denote this by ${ }^{*} T_{x} X$. We call the normal cone $C_{d(X)}(X \times X)$ the tangent star-bundle (not a bundle in the usual sense), and denote it by ${ }^{*} T X$. Thus, $P(X)=\mathbf{P}\left({ }^{*} T X\right)$. Note that if $\Theta(X)=\operatorname{Spec}\left(\operatorname{Sym}_{\mathcal{O}_{X}}\left(I / I^{2}\right)\right)$, then $\Theta_{x} X$ is the Zariski tangent space to $X$ at $x$. The surjection $\operatorname{Sym}_{\mathcal{O}_{X}}\left(I / I^{2}\right) \rightarrow \oplus_{j \geqslant 0} I^{j} / I^{j+1}$ induces a scheme-theoretical inclusion ${ }^{*} T X \hookrightarrow \Theta(X)$, so the tangent star is a subcheme of the Zariski tangent space. Johnson gave a geometric description of the tangent star (as a set) as follows: If $X$ is a subvariety of $\mathbf{C}^{N}$, then ${ }^{*} T_{x} X$ is the union of all lines $L$ 
through $x$ for which there are sequences $\left\{y_{i}\right\},\left\{y_{i}^{\prime}\right\}$ of points in $X$ converging to $x$ such that the sequence of lines $\widetilde{y_{i} y_{i}^{\prime}}$ converges to $L$. In fact, this tangent $\operatorname{star}{ }^{*} T_{x} X$ was already introduced by Whitney as $C_{5}(X, x)$ [Wh, Chapter 7].

Let $A^{N-m-1}$ be a generic linear subspace of $\mathbf{P}^{N}$. Then the projection map $p_{A}$ : $X \rightarrow \mathbf{P}^{m}$, with $A$ as the center of the projection map, induces a linear map $d\left(P_{A}\right)_{x}$ : $\Theta_{x}(X) \rightarrow \Theta_{p_{A}(x)} \mathbf{P}^{m}$ for each point $x \in X$. Johnson defined a ramification locus of $p_{A}$ as follows: $p_{A}$ ramifies at $x$ if the induced map $p_{A} \mid{ }_{*_{T} X} X^{*}{ }^{*} T_{X} X \rightarrow \Theta_{p_{A}(x)} \mathbf{P}^{m}$ is not finite-to-one. He showed that [Jo, Lemma 2.1 and §2.2] $p_{A}$ ramifies at $x$ if and only if $\overline{{ }^{*} T_{X} X} \cap A \neq \varnothing$, where ${ }^{{ }^{*} T_{x} X}$ is the projective closure of the tangent star. The ramification locus is not necessarily equidimensional, but its largest components are of dimension $(2 n-m-1)$ and their union is the support of a scheme $\Re^{A}(X)$ described below.

First we give another description of the ramification locus $R^{A}(X)$. The variety $\overline{X \times X}$ is the closure of the image of the map

$$
X \times X-d(X) \rightarrow(X \times X) \times \mathbf{G}, \quad(x, y) \mapsto(x, y, \widehat{x y}),
$$

where $\mathbf{G}=\operatorname{Gr}(N, 1)$ is the Grassmannian of lines in $\mathbf{P}^{N}$, and $\widehat{x y}$ is the secant line through $x$ and $y$. By definition $P(X)$ is a subscheme of $\overline{X \times X}$. Let $g: P(X) \rightarrow \mathbf{G}$ and $p: P(X) \rightarrow d(X)=X$ be the projection maps. Let $W(H)=\{L \in \mathbf{G} \mid L \cap A$ $\neq \varnothing\}$. Then

$$
R^{A}(X)=p \cdot g^{-1} W(A)
$$

The scheme-theoretical analog of this equation gives the desired ramification scheme

$$
\mathfrak{R}^{A}(X)=p \cdot g^{-1} \mathfrak{B}(A) .
$$

Johnson's ramification class is defined by

$$
R_{k}(X)=p_{*}\left(g^{*}[W(A)] \cap[P(X)]\right), \quad k=m-n+1 .
$$

Its support is the union of the largest components of $R^{A}(X)$, and its homology class is independent of a generic $A$. Johnson [Jo, §5.2] showed the Todd formula

$$
R_{k}(X)=\sum_{i=0}^{k}\left(\begin{array}{c}
n+k \\
i+n
\end{array}\right) U^{k-i} \cap S_{i}(X) .
$$

He also defined a double point class $D_{k-1}(k=m-n+1)$ associated with a generic center $A^{N-m-1}$, and obtained the double point formula

$$
D_{k-1}(X)=U^{k-1} \cap d[X]-\sum_{i=0}^{k-1}\left(\begin{array}{c}
n+k \\
i+n+1
\end{array}\right) U^{k-1-i} \cap S_{i}(X)
$$

where $d$ is the degree of $X^{n} \subseteq \mathbf{P}^{N}$. From the Todd formula and the double point formula, he obtained Johnson's connecting formula:

$$
\operatorname{JCF}(k): U \cdot D_{k-1}-D_{k}=R_{k}, \quad(k=m-n+1) .
$$

This connecting formula implies a quite surprising result: Let $X$ be an $n$-dimensional subvariety of $\mathbf{P}^{N}, N \leqslant 2 n$. If $X$ can be immersed in a lower dimensional projective space by projection, then it can be so embedded. 
One of the intriguing questions about Johnson's Segre classes is their invariance properties in a flat family. Using Johnson's Todd formula one can show that for reduced hypersurfaces $X^{n}$ and $Y^{n}$ of the same degree $d$ in $\mathbf{P}^{n+1}, \operatorname{deg} S_{i}(X)=$ $\operatorname{deg} S_{i}(Y)$ for each $i$. Explicitly, for $i>0$

$$
\begin{aligned}
\operatorname{deg} S_{i}(X)= & (-1)^{i-1}\left(\begin{array}{c}
n+i \\
i-1
\end{array}\right) d^{2} \\
& +(-1)^{i-1}\left\{(i-1)\left(\begin{array}{c}
n+i \\
i
\end{array}\right)-(n+2)\left(\begin{array}{c}
n+i \\
i-1
\end{array}\right)\right\} d
\end{aligned}
$$

3. Decompositions of $P(X)$. In this section we compare the projectivizations $P(X)$ and $\mathbf{P}(\widehat{T X})$ of, respectivley, the tangent star-bundle and the Nash tangent bundle of a projective variety $X$.

Proposition 3.1. For any irreducible projective variety $X$, there exists a canonical morphism $q: \mathbf{P}(\widehat{T X}) \rightarrow P(X)$ such that the image $q(\mathbf{P}(\widehat{T X}))$ is an irreducible component of $P(X)$ and also that $\left.q\right|_{\mathbf{P}\left(\widehat{T X}_{s m}\right)}$ is an isomorphism from $\mathbf{P}\left(\widehat{T X}_{s m}\right)$ into $P\left(X_{s m}\right)$.

Proof. Let $\pi: \operatorname{Gr}\left(T \mathbf{P}^{N}, n\right) \rightarrow \mathbf{P}^{N}$ be the projection map (i.e., Grassmannian bundle map) and $\mathscr{E}$ be the pull-back of the tangent bundle $T \mathbf{P}^{N}$ via $\pi$, i.e., $\mathscr{E}=\pi^{*} T \mathbf{P}^{N}$. Note that the exceptional divisor $E$ of the blowup $\widehat{\mathbf{P}^{N} \times \mathbf{P}^{N}}$ of $\mathbf{P}^{N} \times \mathbf{P}^{N}$ is the projectivization of the tangent bundle $T \mathbf{P}^{N}$, i.e., $E=\mathbf{P}\left(T \mathbf{P}^{N}\right)$, and also that the tautological rank $n$ bundle $E^{n}$ over $\operatorname{Gr}\left(T \mathbf{P}^{N}, n\right)$ is a subbundle of $\mathscr{E}=\pi^{*} T \mathbf{P}^{N}$.

Now consider the following diagram, where $\hookrightarrow$ denotes the inclusion map, and also note that $\nu=\left.\pi\right|_{\hat{X}}$. Then we restrict the map $\mathbf{P}(\mathscr{E}) \rightarrow E$ to $\mathbf{P}(\widehat{T X})$ and denote this restriction map by $q$. If we furthermore restrict $q$ to $\mathbf{P}\left(\widehat{T X}_{s m}\right)$, then we have the isomorphism $\mathbf{P}\left(\widehat{T X}_{s m}\right) \stackrel{\underset{\sim}{\rightarrow}}{\rightarrow} \mathbf{P}\left(X_{s m}\right)$. Hence, since $\mathbf{P}(\widehat{T X})$ is the closure of $\mathbf{P}\left(\widehat{T X}_{s m}\right)$ and $q$ is continuous, $q(\mathbf{P}(\widehat{T X})) \subset P(X)$. Since $q(\mathbf{P}(\widehat{T X}))$ and $\overline{P\left(X_{s m}\right)}$ are irreducible and of the same dimension $(=2 n-1)$, it follows that $q(\mathbf{P}(\widehat{T X}))=\overline{P\left(X_{s m}\right)}$, which is an irreducible component of $P(X)$. Q.E.D.

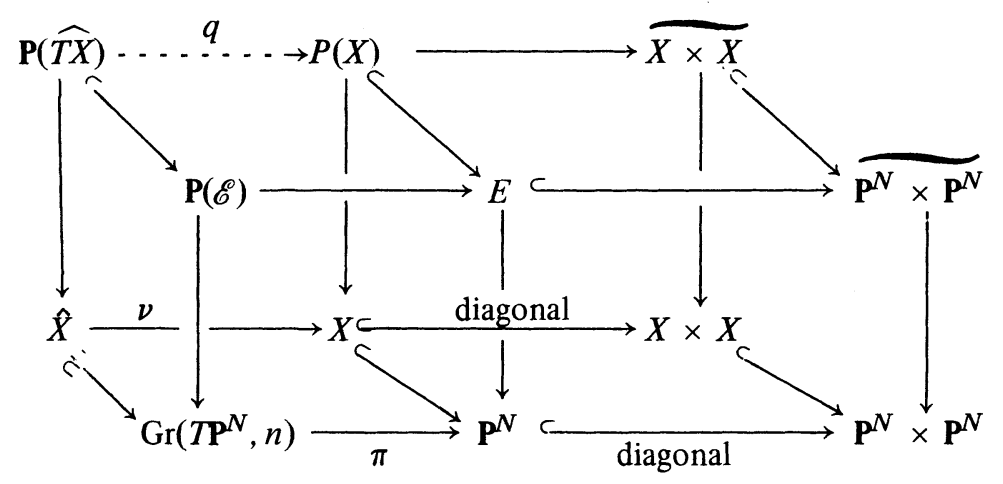

COROLlaRY 3.2. Let $X$ be a reduced projective variety of equidimension and $X=X_{1} \cup \cdots \cup X_{r}$ be the irreducible decomposition of $X$. Then there exists a canonical morphism q: $\mathbf{P}(\widehat{T X}) \rightarrow P(X)$ such that the image $q\left(\mathbf{P}\left(\widehat{T X}_{i}\right)\right)$ is an irreducible 
component of $P(X)$, and that $q(\mathbf{P}(\widehat{T X}))=q\left(\mathbf{P}\left(\widehat{T X}_{1}\right)\right) \cup \cdots \cup q\left(\mathbf{P}\left(\widehat{T X}_{r}\right)\right)$ is the irreducible decomposition of $q(\mathbf{P}(\widehat{T X}))$, and also $q(\mathbf{P}(\widehat{T X}))=\overline{P\left(X_{s m}\right)}$.

We call $q\left(\mathbf{P}\left(\widehat{T X}_{i}\right)\right)$ a typical component of $P(X)$. Note that since $\mathbf{P}(\widehat{T X})$ is reduced and $q$ is an isomorphism, $q(\mathbf{P}(\widehat{T X}))$ is reduced, i.e., the multiplicity of each typical component $q\left(\mathbf{P}\left(\widehat{T X}_{i}\right)\right)$ in $P(X)$ is equal to one.

Now let $\left\{V_{j}\right\}_{j}$ be the extra components of $P(X)$ other than the typical components, supported on the singular part of $X$, with the multiplicity $m_{j}$ for each $V_{j}$, and $\pi_{j}: V_{j} \rightarrow X$ be the projection map. Then it is not hard to show the following naive formula between Johnson's Segre class $S_{*}(X)$ and our Segre-Mather class $S_{*}^{M}(X)$ :

Proposition 3.3. Let $X$ be a reduced projective variety of equidimension $n$. Then we get

$$
S_{i}(X)=S_{i}^{M}(X)+\sum_{j} m_{j} \cdot \pi_{j^{*}}\left(\psi_{j}^{n-1+i} \cap\left[V_{j}\right]\right),
$$

where $\psi_{j}=c_{1}\left(\mathcal{O}_{V_{j}}(1)\right)$.

Proposition 3.4. Let $X^{n} \subset \mathbf{P}^{N}$ be a reduced projective variety of equidimension $n$ and the singular set of $X$ be of dimension $k$. If $N-n<n-k$, then $S_{i}(X)=S_{i}^{M}(X)$ for any $i$.

Proof. Since ${ }^{*} T_{x} X \subseteq T_{x} \mathbf{P}^{N}=\mathbf{C}^{N}$, the "fiber" dimension of each extra component $V_{j}$ of $P(X)$ is at most $N-1$. So the dimension of the extra component $V_{j}$ is at most $(N-1)+k$. Since $N-n<n-k,(N-1)+k<2 n-1$. Hence, in fact, there is no such extra component $V_{j}$ because $P(X)$ must be of equidimension $2 n-1$. Thus the above formula follows from Proposition 3.3. Q.E.D.

REMARK 3.5. The multiplicity $m_{j}$ attached to each extra component $V_{j}$ is given by

$$
m_{j}=\operatorname{length}\left(\mathcal{O}_{P(X), V_{j}}\right) \text {. }
$$

Here $\mathcal{O}_{P(X), V_{j}}$ is the local ring of $P(X)$ at $V_{j}$. As in [Ho1, Proof of Lemma 8.1.1] A. Holme discussed $m_{j}$ a little, and this integral coefficient $m_{j}$ can be interpreted as the intersection multiplicity of the scheme-theoretical intersection $P(X)=E \cap \overline{X \times X}$. This multiplicity is given by Serre's Tor-formula (see [K1, p. 317]):

$$
\sum_{i}(-1)^{i} \text { length }\left(\operatorname{Tor}_{i}^{\mathcal{O}} \widetilde{\mathrm{P} N \times \mathrm{P} N_{N} V_{j}}\left(\mathcal{O} \widetilde{X \times X}, V_{j}, \mathcal{O}_{E, V_{j}}\right)\right) \text {. }
$$

By some algebra this turns out to be equal to length $\left(\mathcal{O}_{P(X), V_{j}}\right)$. It seems that it is hard to compute this multiplicity or even to identify the extra components of $P(X)$. In $\S \S 4$ and 5 we will find such multiplicities in the cases of hypersurfaces and $X^{n} \subseteq \mathbf{P}^{2 n}$ with isolated singularities.

4. Hypersurfaces. In this section we will give an explicit formula between Johnson's Segre class $S_{*}(X)$ and our Segre-Mather class $S_{*}^{M}(X)$ for hypersurfaces without any restrictions on singularities.

Proposition 4.1. Let $X^{n}$ be a reduced hypersurface of $\mathbf{P}^{n+1}$. If $x$ is a singular point of $X$, then the tangent star ${ }^{*} T_{x} X$ to $X$ at $x$ is isomorphic to $T_{x} \mathbf{P}^{n+1}=\mathbf{C}^{n+1}$ (even as a scheme). 
Proof. Since this is a local problem, we can assume that $x \in X \subset \mathbf{C}^{n+1}$, and also we can assume that $x$ is the origin. Let $f\left(X_{1}, X_{2}, \ldots, X_{n+1}\right)$ be a reduced polynomial defining $X$. Since $x$ is a singular point and $x$ is the origin, $f\left(X_{1}, X_{2}, \ldots, X_{n+1}\right)$ has no constant term and the degree of its initial part is $\geqslant 2$. Let $X_{1}, \ldots, X_{n+1}$, $U_{1}, \ldots, U_{n+1}$ be the affine coordinates of $\mathbf{C}^{n+1} \times \mathbf{C}^{n+1}$. Let $J$ be the ideal defining the diagonal $d\left(\mathbf{C}^{n+1}\right)$ of $\mathbf{C}^{n+1} \times \mathbf{C}^{n+1}$, generated by $\left(X_{1}-U_{1}, X_{2}-U_{2}, \ldots, X_{n+1}\right.$ $\left.-U_{n+1}\right), \mathscr{M}=\left(X_{1}, U_{1}, \ldots, X_{n+1}, U_{n+1}\right)$, and $I$ be the ideal defining the diagonal $d(X)$ of $X \times X$ in the coordinate ring

$$
\begin{gathered}
R=\mathbf{C}\left[X_{1}, \ldots, X_{n+1}, U_{1}, \ldots, U_{n+1}\right] /\left(f\left(X_{1}, \ldots, X_{n+1}\right) . f\left(U_{1}, \ldots, U_{n+1}\right)\right), \\
\mathscr{M}=\mathscr{M} /\left(f\left(X_{1}, \ldots, X_{n+1}\right), f\left(U_{1}, \ldots, U_{n+1}\right)\right) .
\end{gathered}
$$

Then by some standard algebra, we have the surjection

$$
\phi: \bigoplus_{j \geqslant 0} J^{j} / \mathscr{M} \cdot J^{j} \rightarrow \bigoplus_{j \geqslant 0} \bar{I}^{j} / \overline{\mathscr{M}} \cdot \bar{I}^{j} .
$$

If $\phi$ is not surjective, i.e. $\operatorname{Ker} \phi$ is not zero, then it follows by taking Proj that

$$
P\left({ }^{*} T_{x} X\right)=\operatorname{Proj}\left(\bigoplus_{j \geqslant 0} \bar{I}^{j} / \overline{\mathscr{M}} \cdot \bar{I}^{j}\right)
$$

is a proper closed subscheme of $\operatorname{Proj}\left(\oplus_{j \geqslant 0} J^{j} / \mathscr{M} \cdot J^{j}\right)=\mathbf{P}\left({ }^{*} T_{x} \mathbf{C}^{n+1}\right)=\mathbf{P}\left(\mathbf{C}^{n+1}\right)=$ $\mathbf{P}^{n}$. Hence $\mathbf{P}\left({ }^{*} T_{x} X\right)$ consists of at most a finite number of hypersurfaces. On the other hand, since the multiplicity of $X$ at $x$ is $\geqslant 2$ (in fact, is equal to the degree of the initial part of the defining polynomial $\left.f\left(X_{1}, \ldots, X_{n+1}\right)\right)$, any line $L$ going through the point is the limit of the secant line $\widehat{x_{i} y_{i}}$, where $x_{i} \rightarrow x, y_{i} \rightarrow x$, and $x_{i}$, $y_{i}$ are smooth points. Thus any line going through the singular point $x$ is in ${ }^{*} T_{x} X$ (as a set); i.e., ${ }^{*} T_{x} X=\mathbf{C}^{n+1}$ as a set, i.e., $\mathbf{P}\left({ }^{*} T_{x} X\right)=\mathbf{P}^{n}$ as a set. This is a contradiction. Thus $\phi$ must be injective, so $\phi$ is an isomorphism. Hence,

$$
\begin{aligned}
& { }^{*} T_{x} X=\operatorname{Spec}\left(\bigoplus_{j \geqslant 0} \bar{I}^{j} / \overline{\mathscr{M}} \cdot \bar{I}^{j}\right) \simeq \operatorname{Spec}\left(\bigoplus_{j \geqslant 0} J^{j} / \mathscr{M} \cdot J^{j}\right) \\
& \left(\underset{\text { def }}{=}\left(* T_{x} C^{n+1}=T_{x} \mathbf{C}^{n+1}\right)\right) \\
& \simeq \operatorname{Spec}\left(\mathbf{C}\left[X_{1}-U_{1}, \ldots, X_{n+1}-U_{n+1}\right]\right) \simeq \mathbf{C}^{n+1} \text {. }
\end{aligned}
$$

Therefore, the tangent $\operatorname{star}{ }^{*} T_{x} X$ to $X$ at a singular point $x$ is $\mathbf{C}^{n+1}=T_{x} \mathrm{C}^{n+1}$ even as a scheme. Q.E.D.

Now we are ready to state our first main theorem.

THEOREM 4.2 (A FORMUla fOR SEgRe ClASSES FOR HYPERSURFACES). Let $X^{n} \subseteq$ $\mathbf{P}^{n+1}$ be a reduced hypersurface with $S$ denoting the singular subvariety of $X$. Then

$$
\begin{aligned}
S_{i}(X) & =S_{i}^{M}(X)+S^{i-1}\left(P^{n+1}\right) \cap\left(\sum_{j} e_{j} \cdot\left[S_{n-1, j}\right]\right) \\
& =S_{i}^{M}(X)+(-1)^{i-1}\left(\begin{array}{c}
n+i \\
i-1
\end{array}\right) U^{i-1} \cap\left(\sum_{j} e_{j} \cdot\left[S_{n-1, j}\right]\right),
\end{aligned}
$$


where $S_{n-1, j}$ are irreducible components of dimension $n-1$ of the singular subvariety $S, e_{j}$ is the multiplicity of the Jacobian ideal in the local ring of $X$ at the generic point of $S_{n-1, j}, U=c_{1}\left(\mathcal{O}_{X}(1)\right)$ and $S^{i-1}\left(\mathbf{P}^{n+1}\right)$ is the usual $(i-1)$ st Segre (cohomology) class of $\mathbf{P}^{n+1}$. Hence, in particular, if $\operatorname{dim} S<n-1$, then $S_{n-1, j}=\varnothing$, so $S_{i}(X)=$ $S_{i}^{M}(X)$ for any $i$.

Proof. The second assertion is clear by Proposition 3.4. So we consider the case when $\operatorname{dim} S=n-1$. In this case, by Proposition 4.1, $P(X)$ has extra components supported on each irreducible component, $S_{n-1, j}$ of dimension $n-1$ of the singular subvariety $S$ of $X$, and they are $\mathbf{P}\left(\left.T \mathbf{P}^{n+1}\right|_{S_{n-1, j}}\right)$. We show the following formula (recalling the notations in §3):

$$
\begin{aligned}
\pi_{j^{*}}\left(\Psi_{j}^{n-1+i} \cap\left[\mathbf{P}\left(\left.T \mathbf{P}^{n+1}\right|_{S_{n-1 . j}}\right)\right]\right) & =S^{i-1}\left(\mathbf{P}^{n+1}\right) \cap\left[S_{n-1, j}\right] \\
& =(-1)^{i-1}\left(\begin{array}{c}
n+i \\
i-1
\end{array}\right) U^{i-1} \cap\left[S_{n-1, j}\right],
\end{aligned}
$$

where we understand $S^{i-1}\left(\mathbf{P}^{n+1}\right)=e^{*} S^{i-1}\left(\mathbf{P}^{n+1}\right), e: X \rightarrow \mathbf{P}^{n+1}$ the inclusion map, and $\left[S_{n-1, j}\right]=i_{j^{*}}\left[S_{n-1, j}\right], i_{j}: S_{n-1, j} \rightarrow X$ the inclusion map.

Proof OF THE AbOVE formula. For convenience, we omit the subscript $j$ from the notation. Then

$$
\left.T \mathbf{P}^{n+1}\right|_{S_{n-1}}=(e \circ i)^{*} T \mathbf{P}^{n+1}=i^{*} e^{*} T \mathbf{P}^{n+1} .
$$

Since $i^{*} e^{*} T \mathbf{P}^{n+1}$ is a rank $(n+1)$ bundle over $S_{n-1}$, by considering $\pi=i \circ \pi^{0}$, where $\pi^{0}: \mathbf{P}\left(\left.T \mathbf{P}^{n+1}\right|_{S_{n-1}}\right) \rightarrow S_{n-1}$ is the projection map, we have

$$
\begin{aligned}
\pi_{*}\left(\psi^{n-1+i} \cap\left[\mathbf{P}\left(\left.T \mathbf{P}^{n+1}\right|_{S_{n-1}}\right)\right]\right)=i_{*} \pi_{*}^{0}\left(\psi^{(n+1)-1+(i-1)} \cap\left[\mathbf{P}\left(\left.T \mathbf{P}^{n+1}\right|_{S_{n-1}}\right)\right]\right) \\
=i_{*}\left(S^{i-1}\left(\left.T \mathbf{P}^{n+1}\right|_{S_{n-1}}\right) \cap\left[S_{n-1}\right]\right)=i_{*}\left(S^{i-1}\left(i^{*} e^{*} T \mathbf{P}^{n+1}\right) \cap\left[S_{n-1}\right]\right) \\
=i_{*}\left(i^{*} e^{*} S^{i-1}\left(T \mathbf{P}^{n+1}\right) \cap\left[S_{n-1}\right]\right)=e^{*} S^{i-1}\left(\mathbf{P}^{n+1}\right) \cap i_{*}\left[S_{n-1}\right] .
\end{aligned}
$$

by the projection formula and the definition of $S^{*}\left(\mathbf{P}^{n+1}\right)$. Since

$$
S\left(\mathbf{P}^{n+1}\right)=1 / C\left(\mathbf{P}^{n+1}\right)=\left(1+c_{1}\left(\mathcal{O}_{\mathbf{P}^{n+1}}(1)\right)\right)^{-(n+2)}
$$

and $e^{*} \mathcal{O}_{\mathbf{p}^{n+1}}(1)=\mathcal{O}_{X}(1)$, by a well-known binomial formula

$$
e^{*} S^{i-1}\left(\mathbf{P}^{n+1}\right)=(-1)^{i-1}\left(\begin{array}{l}
n+i \\
i-1
\end{array}\right) U^{i-1} .
$$

Thus, by Proposition 3.3, we get the following formula:

$$
S_{i}(X)=S_{i}^{M}(X)+S^{i-1}\left(\mathbf{P}^{n+1}\right) \cap\left(\sum_{j} m_{j} \cdot\left[S_{n-1, j}\right]\right) .
$$

So all we have to do is to find $\sum_{j} m_{j} \cdot\left[S_{n-1, j}\right]$ (or strongly $m_{j}$ for each $j$ ).

Now by Johnson's Todd formula and our Todd formula relating $\bar{P}_{*}(X)$ and $S_{*}^{M}(X)$ we easily get

$$
R_{1}(X)=\bar{P}_{1}(X)+\sum_{j} m_{j} \cdot\left[S_{n-1, j}\right]
$$


Recalling the definitions of $P_{*}(X)$ and $\bar{P}_{*}(X)$ in $\$ 1$ we notice that $\bar{P}_{1}(X)=P_{1}(X)$. (In general, of course, $\bar{P}_{k}(X) \neq P_{k}(X)$.) By [Pi1, Corollary 2.2, p. 257]

$$
P_{1}(X)=(d-1) U \cap[X]-\sum_{j} e_{j} \cdot\left[S_{n-1, j}\right],
$$

where $d$ is the degree of the hypersurface $X$. Johnson's double point formula implies that $D_{0}(X)=d[X]-[X]=(d-1)[X]$ and also Johnson's connecting formula implies that, since $D_{1}(X)=0$ (because $X$ is already contained in $\mathbf{P}^{n+1}$ ), $R_{1}(X)=U$ $\cap D_{0}(X)$. Then we combine them to get

$$
R_{1}(X)=(d-1) U \cap[X] .
$$

Thus (4.4) and (4.5) imply

$$
R_{1}(X)=P_{1}(X)+\sum_{j} e_{j} \cdot\left[S_{n-1, j}\right]
$$

Thus (4.3) and (4.6) imply

$$
\sum_{j} m_{j} \cdot\left[S_{n-1, j}\right]=\sum_{j} e_{j} \cdot\left[S_{n-1, j}\right] .
$$

This completes the proof of the theorem.

REMARK 4.8. One might be tempted to immediately conclude that for each $j$, $m_{j}=e_{j}$. But identity (4.7) is in the homology group (or Chow homology group), thus, as cycles, $\Sigma_{j}\left(m_{j}-e_{j}\right) \cdot\left[S_{n-1, j}\right]$ is homologous to zero (or rationally equivalent to zero). If the singular locus of $X$ has only one irreducible component of dimension $n-1$, then $m=e$; i.e., $e=\operatorname{length}\left(\mathcal{O}_{P(X), V}\right)$. Also, both $m$ and $e$ are described locally, so we conjecture that $m_{j}=e_{j}$ for each $j{ }^{1}$

REMARK 4.9. As an example of Theorem 4.2, let us consider a reduced plane curve $X$ with isolated singularities $x_{1}, \ldots, x_{r}$. Then we have

$$
S_{1}(X)=S_{1}^{M}(X)+\sum_{j} e_{i}\left[x_{i}\right]
$$

where $e_{i}$ is the Jacobian multiplicity of $x_{i}$. For instance the Jacobian multiplicity of a node singularity is 2 and that of a cusp singularity is 3 (see [KI]). This formula and Dubson's formula for Chern-MacPherson classes $C_{*}(X)$ give an interesting formula between $S_{1}(X)$ and $C_{1}(X)$ involving Milnor numbers:

$$
S_{1}(X)=-C_{1}(X)+\sum_{i} \mu_{i}\left[x_{i}\right]
$$

where $\mu_{i}$ is the Milnor number of the singularity $x_{i}$. We have been unable to generalize this to other higher dimensional cases.

5. $X^{n} \subseteq \mathbf{P}^{2 n}$ with isolated singularities. In $\$ 4$ we considered the hypersurface case, a special case of which is the plane curve case. In this section we consider the case when $X^{n} \subseteq \mathbf{P}^{2 n}$ with isolated singularities, a special case of which is also the plane curve case.

\footnotetext{
${ }^{1} R$. Varley and the present author have recently proved this conjecture affirmatively. The proof will appear elsewhere.
} 
If $X^{1} \subset \mathbf{P}^{2}$ is a reduced plane curve of degree $d$ with a single singularity $x_{0}$, then we have

$$
S_{0}(X)=S_{0}^{M}(X)=[X] \quad \text { and } \quad S_{1}(X)=S_{1}^{M}(X)+m \cdot\left[x_{0}\right],
$$

where $m$ is the Jacobian multiplicity of $x_{0}, m=d(d-1)-\operatorname{deg}\left(\bar{P}_{1}(X)\right)$. This can be generalized to

THEOREM 5.1. Let $X^{n} \subseteq \mathbf{P}^{2 n}$ be a reduced singular variety of degree $d$ with a single isolated singularity $x_{0}$. Then

$$
S_{i}(X)=S_{i}^{M}(X), \quad i<n, \quad \text { and } \quad S_{n}(X)=S_{n}^{M}(X)+m \cdot\left[x_{0}\right],
$$

where

$$
m=d(d-1)-\sum_{k=1}^{n} \operatorname{deg}\left(\bar{P}_{n-k+1}(X)\right) .
$$

Proof. The first equality is clear (by the dimension reason). We note that

$$
S_{n}(X)=S_{n}^{M}(X)+m \cdot\left[x_{0}\right],
$$

where $m$ is a nonnegative integer. By Johnson's Todd formula and our Todd formula (see $\S 1$ ) we can easily see that

$$
R_{n-k+1}(X)=\bar{P}_{n-k+1}(X), \quad 2 \leqslant k \leqslant n,
$$

and

$$
R_{n}(X)=\bar{P}_{n}(X)+m \cdot\left[x_{0}\right] .
$$

Then by Johnson's connecting formula, (5.2), and (5.3) we have

$$
\begin{array}{lll}
\operatorname{JCF}(1) & U \cdot D_{0}-D_{1} & =\bar{P}_{1}, \\
& & \vdots \\
\operatorname{JCF}(k) & U \cdot D_{k-1}-D_{k} & =\bar{P}_{k}, \\
& \vdots \\
\operatorname{JCF}(n-1) & U \cdot D_{n-2}-D_{n-1} & =\bar{P}_{n-1}, \\
\operatorname{JCF}(n) . & U \cdot D_{n-1}-D_{n} & =\bar{P}_{n}+m\left[x_{0}\right] .
\end{array}
$$

Now by applying $U^{n-k}$ on both sides of each $\operatorname{JCF}(k)$ and adding all of $U^{n-k}$. $\operatorname{JCF}(k), 1 \leqslant k \leqslant n$, we obtain

$$
U^{n} \cdot D_{0}-D_{n}=\sum_{k=1}^{n} U^{k-1} \cdot \bar{P}_{n-k+1}(X)+m \cdot\left[x_{0}\right] .
$$

Since $X^{n} \subseteq \mathbf{P}^{2 n}, D_{n}=0$, so (5.4) becomes

$$
U^{n} \cdot D_{0}-\sum_{k=1}^{n} U^{k-1} \cdot \bar{P}_{n-k+1}(X)=m \cdot\left[x_{0}\right] .
$$

Since $D_{0}=(d-1)[X],(5.5)$ becomes

$$
(d-1) U^{n} \cdot[X]-\sum_{k=1}^{n} U^{k-1} \bar{P}_{n-k+1}(X)=m \cdot\left[x_{0}\right] .
$$

Since $X^{n}$ is of dimension $n$ and $\bar{P}_{n-k+1}(X)$ is of dimension $(k-1)$, we obtain

$$
d(d-1)-\sum_{k=1}^{n} \operatorname{deg}\left(\bar{P}_{n-k+1}(X)\right)=m .
$$

This completes the proof of the theorem. 
REMARK 5.2. In general, if $X^{n} \subseteq \mathbf{P}^{N}(N \geqslant 2 n)$ with a single isolated singularity, then the multiplicity $m$ becomes

$$
m=d(d-1)-\operatorname{deg}\left(D_{n}(X)\right)-\sum_{k=1}^{n} \operatorname{deg}\left(\bar{P}_{n-k+1}(X)\right) .
$$

As for $\operatorname{deg}\left(D_{n}(X)\right)$, refer to [HR, Theorem 3.3 and Ho2].

Theorem 5.1 is valid only for the single isolated singularity case. If a singular variety has many isolated singularities, say $x_{1}, x_{2}, \ldots, x_{r}$, then as a corollary of the proof of Theorem 5.1, we have

$$
\sum_{i=1}^{r} m_{i}=d(d-1)-\sum_{k=1}^{n} \operatorname{deg}\left(\bar{P}_{n-k+1}(X)\right),
$$

where

$$
\text { (\#) } \quad m_{i}= \begin{cases}\text { length }\left(\mathcal{O}_{P(X), V_{i}}\right), & \text { where } V_{i}=\mathbf{P}\left(* T_{x_{i}} X\right) \text { is supported on } \\ & \text { the singularity } x_{i} \text { and } \operatorname{dim}\left({ }^{*} T_{x_{i}} X\right)=2 n, \\ 0, & \text { if } \operatorname{dim}\left(* T_{x_{i}} X\right)<2 n\end{cases}
$$

and

$$
S_{n}(X)=S_{n}^{M}(X)+\sum_{i=1}^{r} m_{i}\left[x_{i}\right]
$$

or, equivalently,

$$
R_{n}(X)=\bar{P}_{n}(X)+\sum_{i=1}^{r} m_{i}\left[x_{i}\right] .
$$

By (\#), if we use Fulton's notation (see $\S 6.1$ of [F4] and a remark right after Lemma 7.1, p. 120), we can see

Proposition 5.3. Under the same hypothesis as above,

$$
m_{i}\left[x_{i}\right]=\left(\mathbf{P}^{2 n} \cdot(X \times X)\right)^{\left\{\left(x_{i}, x_{i}\right)\right\}},
$$

which is called the part of $\mathbf{P}^{2 n} \cdot(X \times X)$ supported on $\left(x_{i}, x_{i}\right)$.

We may call this the localized self-intersection class of $X$ at $x_{i}$ and for simplicity denote it by $(X \cdot X)^{\left\{x_{i}\right\}}$. So we can express $\left(5.1^{\prime}\right)$ as follows:

$$
S_{n}(X)=S_{n}^{M}(X)+\sum_{i=1}^{r}(X \cdot X)^{\left\{x_{i}\right\}} .
$$

Now, just as in the hypersurface case (cf. Remark 4.8) we will give the integer $m_{i}=$ length $\left(\mathcal{O}_{P(X), V_{i}}\right)$ (or $=0$ ) a local (and "down-to-earth") description with respect to the singular point $x_{i}$. For this we introduce the following definitions.

Definition 5.4. Let $P$ be a point in $\mathbf{P}^{2 n}$ and let $H$ be a hyperplane not containing the point $P$. We identify $\mathbf{P}^{2 n}-H$ with $\mathbf{C}^{2 n}$. Let $t$ be a complex number. Then a shift $S_{P, t}(y)$ of a point $y(\neq P) \in \mathbf{C}^{2 n}$ is defined to be (see Figure 1)

$$
S_{P, t}(y)=y+t \cdot \frac{P-y}{\|P-y\|},
$$


where $\|P-y\|$ is the norm of the vector $P-y$, i.e.,

$$
P-y=\sqrt{\left|P_{1}-y_{1}\right|^{2}+\cdots+\left|P_{2 n}-y_{2 n}\right|^{2}}
$$

if we let $P=\left(P_{1}, \ldots, P_{2 n}\right)$ and $y=\left(y_{1}, \ldots, y_{2 n}\right)$.

Definition 5.5. Let $X^{n} \subseteq \mathbf{P}^{2 n}$ with isolated singularities. Let $x$ be a singular point and $P$ be a point off $X$. Let $H$ be hyperplane not containing $x$ and consider the affine variety $X^{a}=X-H \subset \mathbf{P}^{2 n}-H=\mathbf{C}^{2 n}$. We assume that $P$ does not lie on $H$. Let $\varepsilon$ be a small postiive number and $t$ a complex number such that $|t| \ll \varepsilon$, and $B_{\varepsilon}(x)$ an $\varepsilon$-ball around $x$. Then the number $n(x, P, t, \varepsilon)$ associated to the singular point $x$ is defined to be (see Figure 2) \#( $S_{P, t}\left(X^{a}\right) \cap X^{a} \cap B_{\varepsilon}(x)$ ), if $S_{P, t}\left(X^{2}\right)$ is transverse to $X^{a}$ within $B_{\varepsilon}(x)$. (We will see later that $S_{P, t}\left(X^{a}\right)$ and $X^{a}$ are transverse to each other within $B_{\varepsilon}(x)$ for a generic point $P$ off $X$ and sufficiently small $\varepsilon$ and $t$.)

We note that as long as we look at a sufficiently small neighborhood of $x$, we may consider the shift $S_{P, t}$ as the translation via the direction vector $t \cdot(P-x) /\|P-x\|$.

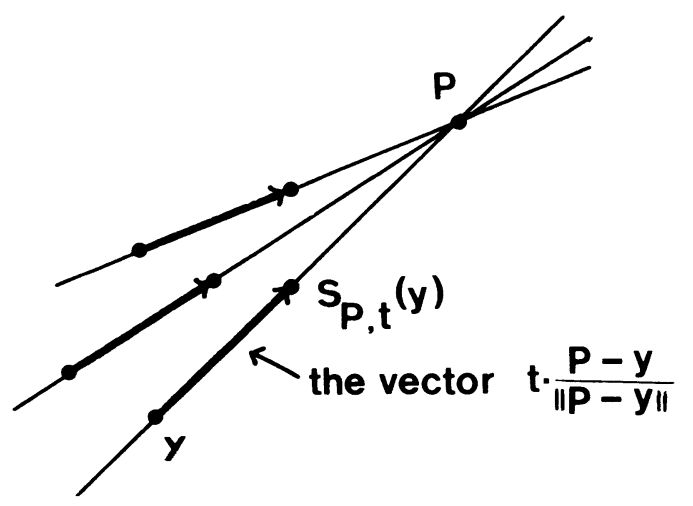

Figure 1

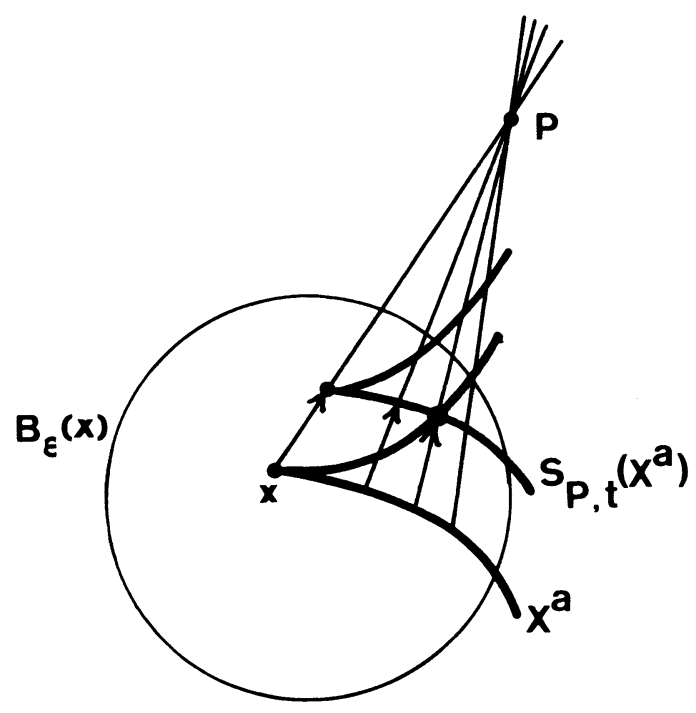

Figure 2 
Now we are ready to state the local description of the integer $m_{i}$ appearing in $\left(5.1^{\prime}\right)$ or $\left(5.3^{\prime}\right)$.

THEOREM 5.6. Let $X^{n} \subseteq \mathbf{P}^{2 n}$ be a reduced singular variety with isolated singularities $x_{1}, x_{2}, \ldots, x_{r}$. Let $P, t, \varepsilon$ be as in Definition 5.5. Then

(i) For each singularity $x_{i}$, the number $n_{i}=n\left(x_{i}, P, t, \varepsilon\right)$ is well defined and constant for a generic point $P$,

(ii) The integer $m_{i}$ appearing in $\left(5.1^{\prime}\right)$ is exactly equal to $n_{i}$, i.e.,

$$
S_{n}(X)=S_{n}^{M}(X)+\sum_{i=1}^{r} n_{i}\left[x_{i}\right]
$$

Proof. Since $\left(5.1^{\prime}\right)$ and $\left(5.3^{\prime}\right)$ are equivalent and the objects of Johnson's ramification class $R_{n}(X)$ and our polar class $\bar{P}_{n}(X)$ are more geometrical, we look at $\left(5.3^{\prime}\right)$ instead of $\left(5.1^{\prime}\right)$. And the idea (or strategy) of our proof is as follows: First we take a closer and more careful look at Johnson's ramification cycle, whose homology class (or rational equivalence class) is Johnson's ramification class, and then step by step we recapture the multiplicity $m_{i}$ within a more tractable or "down-to-earth" set up. We prove both (i) and (ii) simultaneously.

First, for the sake of convenience, we set up the following notations:

$B L=\widetilde{\mathbf{P}^{\mathbf{N}} \times \mathbf{P}^{\mathbf{N}}}$, the blowup of $\mathbf{P}^{\mathbf{N}} \times \mathbf{P}^{\mathbf{N}}$ along the diagonal,

$E=$ the exceptional divisor of $B L$,

$\pi: \overline{X \times X} \rightarrow X \times X$, the blowup map,

$\gamma: \overline{X \times X} \rightarrow \mathbf{G}=\operatorname{Gr}(N, 1)$, the Gauss map,

$W(p)=\{L \in \mathbf{G} \mid L \cap P \neq \varnothing\}$, the first Schubert variety, where $P$ is a generic center (in our case, $P$ is a point),

$i: \overline{X \times X} \rightarrow B L$, the inclusion map,

$j: \tilde{D}=\tilde{D}(X) \rightarrow \overline{X \times X}$, the inclusion map, where $\tilde{D}=\tilde{D}(X)=\gamma^{-1}(W(P))$ is the double point scheme,

$\tilde{R}=\tilde{R}(X)=g^{-1}(W(P))$, the ramification scheme, where $g=\left.\gamma\right|_{P(X)}$ is the restriction of $\gamma$ to $P(X)$,

$k: P(X) \rightarrow \widehat{X \times X}$, the inclusion map,

$p: P(X) \rightarrow X$, the projection map defined by $p=\operatorname{pr}_{1} \circ \pi \circ k$, where $\operatorname{pr}_{1}: X \times X$ $\rightarrow X$ is the projection map.

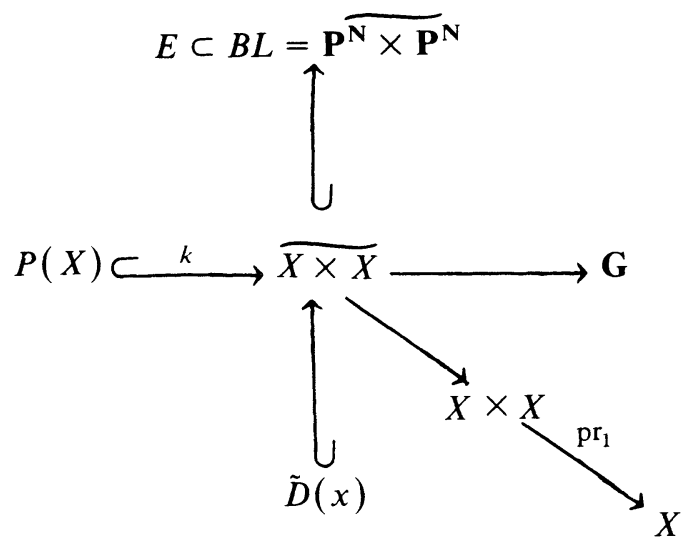


Then, Johnson's ramification cycle $R_{n}(X)$ is, by definition,

$$
\begin{aligned}
R_{n}(X) & =p_{*} g^{*}[W(P)] \quad \text { (using Fulton's notation) } \\
& =p_{*}\left(g^{*}[W(P)] \cap[P(X)]\right) \\
& \left.=\operatorname{pr}_{1^{*}} \cdot \pi_{*} \cdot k_{*}\left(g^{*}[W(P)] \cap[P(X)]\right) \quad \text { (because } p=\operatorname{pr}_{1} \circ \pi \circ k\right) .
\end{aligned}
$$

Since $\tilde{R}=g^{-1}(W(P))$,

$$
\begin{aligned}
{[\tilde{R}] } & =g^{*}[W(P)] \quad \text { (using Fulton's notation) } \\
& =g^{*}[W(P)] \cap[P(X)] .
\end{aligned}
$$

Thus

Let

$$
R_{n}(X)=p_{*}[\tilde{R}]=\operatorname{pr}_{1^{*}} \cdot \pi_{*} \cdot k_{*}[\tilde{R}]
$$

$$
[\tilde{R}]=\sum_{i=1}^{r} n_{i}\left[\left(x_{i}, x_{i}, L_{x_{i}}\right)\right]+\sum_{j=r+1}^{s} n_{j}\left[\left(x_{j}, x_{j}, L_{x_{j}}\right)\right]
$$

where

(1) Each $x_{i}(1 \leqslant i \leqslant r)$ is a singular point, and each $L_{x_{i}}$ is a line in the projective tangent star ${ }^{*} T_{x_{i}} X$ such that $L_{x_{i}}$ hits the generic center $P \cdot n_{i}$ attached to each $\left[\left(x_{i}, x_{i}, L_{x_{i}}\right)\right]$ is a multiplicity of $\left(x_{i}, x_{i}, L_{x_{i}}\right)$ in the ramification scheme $\tilde{R}$. Here if $\operatorname{dim}{ }^{*} T_{x_{i}} X<2 n$ for a singular point $x_{i}$, then by the genericity of the center $P,{ }^{*} T_{x_{i}} X$ misses the point $P$, hence $\left[\left(x_{i}, x_{i}, L_{x_{i}}\right)\right]$ does not appear in $[\tilde{R}]$. For this case we set $n_{i}=0$.

(2) Each $x_{j}(r+1 \leqslant j<s)$ is a polar point and a smooth point (by the genericity of the center $P$ ) and each $L_{x_{j}}$ is in the projective tangent space $\overline{T_{x_{j}} X}$.

Then we can show

LEMMA 5.7. (1) $n_{j}(r+1 \leqslant j \leqslant s)$ is equal to 1 ,

(2) $n_{i}(1 \leqslant i \leqslant r)$ is equal to $m_{i} k_{i}$ for some positive integer $k_{i}$.

Proof. (1) If we restrict $g: P(X) \rightarrow \operatorname{Gr}(N, 1)$ to the reduced subscheme $q(\mathbf{P}(\widehat{T X}))$ of $P(X)$ (see $\S 3$ ) and let $\hat{g}$ be the restriction map, then for a generic point $P$ $\hat{g}^{-1}(W(P))$ is the polar point (cf. [Jo, Proof of Theorem of §3.2]), i.e., as a cycle

$$
\hat{g}^{-1}(W(P))=\sum_{j=r+1}^{s} n_{j}\left[\left(x_{j}, x_{j}, L_{x_{j}}\right)\right] .
$$

However, by [Pi1, Lemma (1.3)], $\hat{\mathrm{g}}^{-1}(W(P))$ is reduced for a generic point $P$, hence each $n_{j}$ must be equal to 1 .

(2) The extra components of $P(X)$ are $\mathbf{P}\left({ }^{*} T_{x_{i}} X\right)$ with the multiplicity $m_{i}$ (see (\#) before Proposition 5.3) and if we restrict $g: P(X) \rightarrow \operatorname{Gr}(N, 1)$ to these components, then we obtain the ramification (but not polar) cycle

$$
\sum_{i=1}^{r} n_{i}\left[\left(x_{i}, x_{i}, L_{x_{i}}\right)\right]
$$

Thus $n_{i}$ is a positive multiple of $m_{i}$, i.e.,

$$
n_{i}=m_{i} \cdot k_{i} \text { for some integer } k_{i} \geqslant 1 .
$$

This completes the proof of the lemma. 
Since $k$ is the inclusion map and, by Lemma 5.7 ,

$$
\begin{aligned}
{[k(\tilde{R})]=} & k_{*}[\tilde{R}]=\sum_{i=1}^{r} m_{i} \cdot k_{i}\left[\left(x_{i}, x_{i}, L_{x_{i}}\right)\right] \\
& +\sum_{j=r+1}^{s}\left[\left(x_{j}, x_{j}, L_{x_{j}}\right)\right] .
\end{aligned}
$$

Now, since $\pi$ is a one-to-one map on the reduced scheme (i.e., variety), $k(\tilde{R})_{\text {red }}=$ $\left\{\left(x_{i}, x_{i}, L_{x_{i}}\right)\right\}_{i=1}^{s}$ and $\pi\left(\left(x_{i}, x_{i}, L_{x_{i}}\right)\right)=\left(x_{i}, x_{i}\right)$, by [F3, §3.1],

$$
\pi_{*} \cdot k_{*}[\tilde{R}]=\sum_{i=1}^{r} m_{i} \cdot k_{i}\left[\left(x_{i}, x_{i}\right)\right]+\sum_{j=r+1}^{s}\left[\left(x_{j}, x_{j}\right)\right] \text {. }
$$

Similarly, since $\mathrm{pr}_{1}$ is a one-to-one map on the diagonal

$$
R_{n}(X)=\operatorname{pr}_{1^{*}} \cdot \pi_{*} \cdot k_{*}[\tilde{R}]=\sum_{i=1}^{r} m_{i} \cdot k_{i}\left[x_{i}\right]+\sum_{j=r+1}^{s}\left[x_{j}\right] .
$$

Since $\sum_{j=r+1}^{s}\left[x_{j}\right]$ is the polar part, i.e., $\bar{P}_{n}(X)=\sum_{j=r+1}^{s}\left[x_{j}\right]$,

$$
R_{n}(X)=\bar{P}_{n}(X)+\sum_{i=1}^{r} m_{i} \cdot k_{i}\left[x_{i}\right]
$$

Then (5.9) and (5.3') imply

$$
\sum_{i=1}^{r} m_{i} \cdot k_{i}\left[x_{i}\right]=\sum_{i=1}^{r} m_{i}\left[x_{i}\right]
$$

i.e.,

$$
\sum_{i=1}^{r} m_{i}\left(k_{i}-1\right)\left[x_{i}\right]=0 .
$$

Since $m_{i}$ is positive, $k_{i}=1$, i.e., $n_{i}=m_{i}$. Hence, by (5.8), we have

OBSERVATION 5.8. The integer $m_{i}=\operatorname{length}\left(\mathcal{O}_{P(X), V_{i}}\right)$ is nothing but the multiplcity $n_{i}$ appearing in the ramification scheme $k(\tilde{R})$ in $\overline{X \times X}$.

LEMMA 5.9. Let $h=i \circ j: \tilde{D} \rightarrow B L$. Then using Fulton's notation, we get

$$
k_{*}[R]=j_{*} h^{*}[E] \text {. }
$$

Proof.

$$
\begin{aligned}
k_{*}[R] & =k_{*}\left(g^{*}[W(P)] \cap[P(X)]\right) \\
& =k_{*}\left(k^{*} \circ \gamma^{*}[W(P)] \cap[P(X)]\right) \quad \text { because } g=\gamma \circ k, \\
& =\gamma^{*}[W(P)] \cap k_{*}[P(X)] \quad \text { by the projection formula. }
\end{aligned}
$$

Using the following identities (cf. [Jo, Appendix B])

$$
\begin{aligned}
& k_{*}[P(X)]=i^{*}(E) \cap[\overline{X \times X}], \\
& j_{*}[\tilde{D}]=\gamma^{*}[W(P)] \cap[\overline{X \times X}],
\end{aligned}
$$


we get

$$
\begin{aligned}
k_{*}[\tilde{R}] & =\gamma^{*}[W(P)] \cap\left(i^{*}[E] \cap[\overline{X \times X}]\right) \\
& =i^{*}[E] \cap\left(\gamma^{*}[W(P)] \cap[\overline{X \times X}]\right) \\
& =i^{*}[E] \cap j_{*}[\tilde{D}] \\
& =j_{*}\left(j^{*} i^{*}[E] \cap[\tilde{D}]\right) \text { by the projection formula } \\
& =j_{*}\left(h^{*}[E] \cap[\tilde{D}]\right) \quad \text { because } h=i \circ j \\
& =j_{*} h^{*}[E] \quad \text { using Fulton's notation. }
\end{aligned}
$$

Thus we get (5.11).

Since $j$ is an embedding, the multiplicity $n_{i}$ appearing in $k_{*}[\tilde{R}]$ is nothing but the multiplicity appearing in $h^{*}[E]$. Therefore we only have to analyze $h^{*}[E]$, i.e., to analyze the scheme-theoretical intersection $h(\tilde{D})$ and $E$, because $h$ is an embedding. (See Figure 3.)

For this analysis, first of all, we observe the following two things: (1) There is a projective line bundle over $B L$, denoted by $K$, which is the pull-back of the tautological bundle over $\mathbf{G}=\operatorname{Gr}(N, 1)$ via the Gauss map $B L \rightarrow \mathbf{G}$. To be more precise, let

$$
q: \mathbf{P}^{N} \times \underline{\mathbf{P}}^{N}-d\left(\mathbf{P}^{N}\right) \rightarrow \mathbf{P}^{N} \times \mathbf{P}^{N} \times G
$$

be defined by $g(x, y)=(x, y, \widehat{x y})$, where $\widehat{x y}$ is the projective line going through the two points $x$ and $y$. Then $\mathrm{BL}$ is the closure of the image $q\left(\mathbf{P}^{N} \times \mathbf{P}^{N} \backslash d\left(\mathbf{P}^{N}\right)\right)$. Let $p: B L \rightarrow \mathbf{G}$ be the restriction of the canonical projection map $p_{3}: \mathbf{P}^{N} \times \mathbf{P}^{N} \times \mathbf{G} \rightarrow$ $\operatorname{Gr}(N, 1)$. Let $\check{K}$ be the tautological $\mathbf{P}^{1}$-bundle over $\mathbf{G}$, which is a subbundle of the trivial $\mathbf{P}^{N}$-bundle over $\mathbf{G}$. Then the bundle $K$ over $B L$ is defined to be the pull-back $p^{*} \check{K}$ of $\check{K}$ via the map $p: B L \rightarrow \mathbf{G}$. So, $K$ is a subbundle of the trivial $\mathbf{P}^{N}$-bundle over $B L$, which is $p^{*}\left(\mathbf{P}^{N} \times \mathbf{G}\right)$.

(2) Then we can define the two canonical sections $s_{1}, s_{2}: B L \rightarrow K$ as follows:

$$
s_{1}((x, y, \text { line }))=((x, y, \text { line }), x), \quad s_{2}((x, y, \text { line }))=((x, y, \text { line }), y) .
$$

$\left(\right.$ Clearly $s_{1}(B L) \cap s_{2}(B L)=s_{1}(E)=s_{2}(E)$.)

Now, our procedure of the analysis is as follows: (Step 1) Capture the schemetheoretical intersection of $h(\tilde{D})$ and $E$ as the scheme-theoretical intersection of $s_{2} h(\tilde{D})$ and $s_{1}(B L)$, and (Step 2) finally, get the local description for the multiplicity $n_{i}$ described in statement (i) in the theorem. (See Figure 4.)
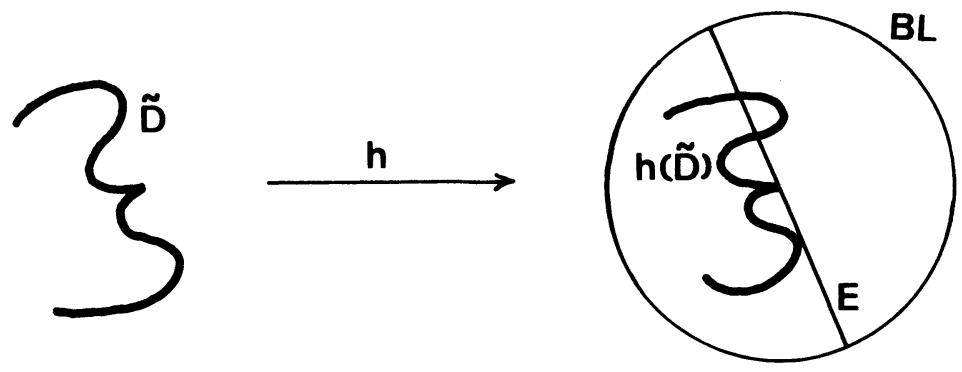

Figure 3 

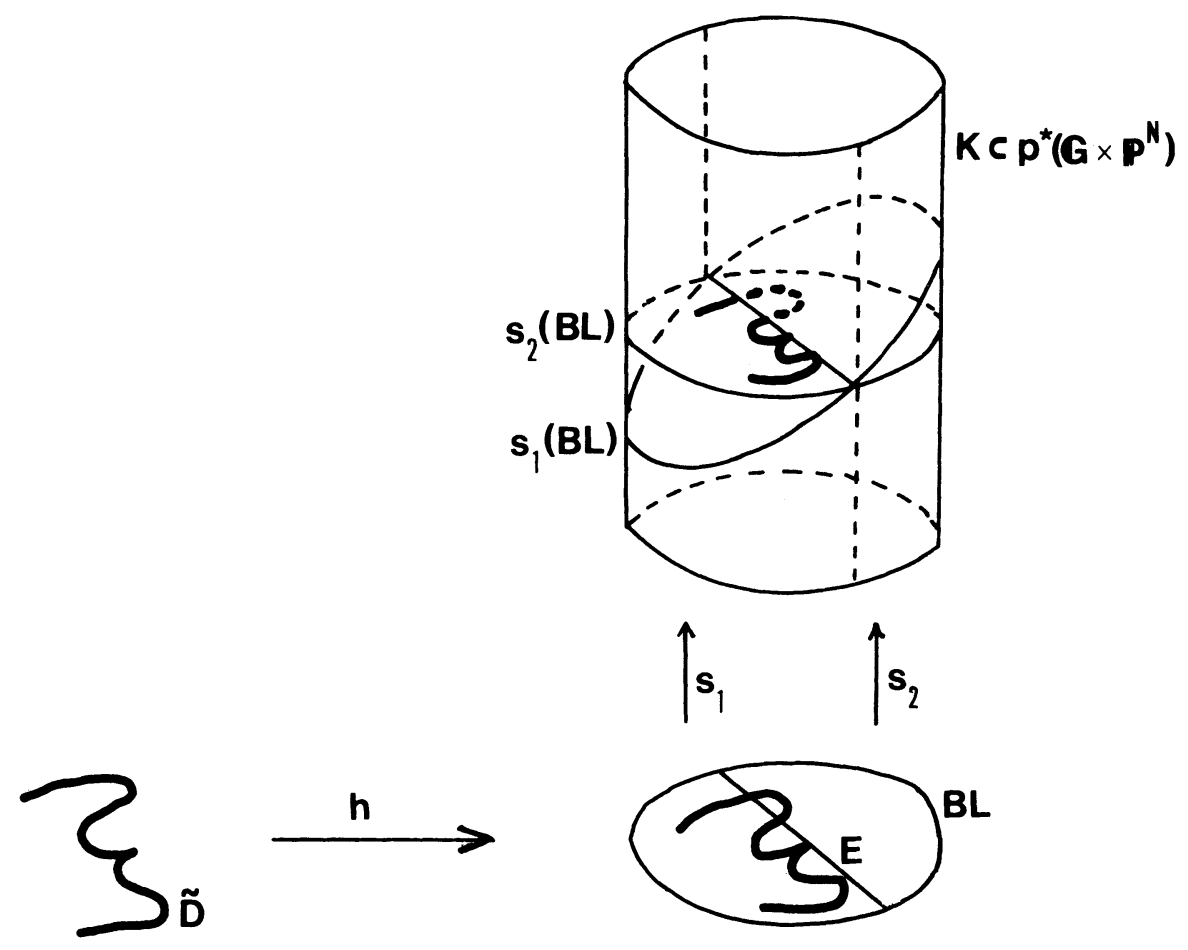

Figure 4

Step 1. It is not hard to show that $s_{1}$ and $s_{2}$ are transverse, i.e., that $s_{1}(B L)$ and $s_{2}(B L)$ are transverse. Then we get

LEMMA 5.10. $h^{*}[E]=\left(s_{2} h\right)^{*}\left[s_{1}(B L)\right]$, using Fulton's notation.

Proof. Since $s_{1}$ and $s_{2}$ are transverse, and $B L$ is reduced,

$$
s_{2}^{-1} s_{1}(B L)=s_{2}^{-1} s_{1}(B L)_{\text {red. }}
$$

It is clear that $s_{2}^{-1} s_{1}(B L)_{\text {red }}=E_{\text {red }}$ (as a set). Since $E$ is also reduced, we get $s_{2}^{-1} s_{1}(B L)=E$, as schemes. Hence

$$
\begin{aligned}
h^{*}[E] & =h^{*}\left[s_{2}^{-1} s_{1}(B L)\right] \\
& =h^{*} s_{2}^{*}\left[s_{1}(B L)\right] \text { using Fulton's notation } \\
& =\left(s_{2} h\right)^{*}\left[s_{1}(B L)\right] .
\end{aligned}
$$

Therefore, since $s_{2} h: \tilde{D} \rightarrow K$ is a scheme-theoretical embedding, $\left(s_{2}\right)^{*}\left[s_{1}(B L)\right]$ is considered as the scheme-theoretical intersection of $s_{2} h(\tilde{D})$ and $s_{1}(B L)$ (cf. Figure 4).

Step 2. Now we are going to show that the multiplicity $m_{i}$ of the point $\left(x_{i}, x_{i}, L_{x_{i}}, x_{i}\right)$ is the scheme-theoreitcal intersection of $s_{2} h(\tilde{D})$ and $s_{1}(B L)$ is equal to $n\left(x_{i}, P, t, \varepsilon\right)$ described in Definition 5.5.

Since in our case $\tilde{D}$ has dimension $1=2 n-(2 n-1)($ see $[\mathbf{J o}]), \operatorname{dim} B L=2 N$ and $\operatorname{dim}\left(s_{2} h(\tilde{D}) \cap s_{1}(B L)=0, s_{2} h(\tilde{D})\right.$ and $s_{1}(B L)$ intersect properly. Also we note that $s_{1} h(\tilde{D})$ and $s_{1}(B L)$ are reduced, i.e., that $\tilde{D}$ and $B L$ are reduced. The 
reducedness of $B L$ is clear. As for the reducedness of $\tilde{B}$, we consider two cases: if $n>1$, then $2 n-1>n$; i.e., $X^{n}$ is projected to the projective space $\mathbf{P}^{2 n-1}$ whose dimension is strictly greater than the dimension of $X^{n}$. In this case, it is known (see $\left[K 1\right.$, p. 383]) that $\operatorname{pr}_{1^{*}} \pi_{*}[\tilde{D}]=[D]$ has no multiple components; hence $\tilde{D}$ must be reduced, or else [ $D$ ] has a multiple component. If $n=1$, i.e., in the planc curve case, $[D]=\operatorname{pr}_{1^{*}} \pi_{*}[\tilde{D}]=(d-1)[X]$, where $d$ is the degree of $X$, and $\tilde{D}$ is a $(d-1)$ sheeted cover of $X$, so $\tilde{D}$ must be reduced, otherwise $\operatorname{pr}_{*} \pi_{*}[D]=(d-1)[X]$ does not hold. Thus in any case, $D$ is a reduced scheme, so $s_{2} h(\tilde{D})$ is reduced. Then it is known (see [FM2, §1 and §4]) that this scheme-theoretical intersection multiplicity $m_{i}$ is nothing but the topological intersection multiplicity of $s_{2} h(\tilde{D})$ and $s_{1}(B L)$ at the point $\left(x_{i}, x_{i}, L_{x_{i}}, x_{i}\right)$. For this we move $s_{1}(B L)$ slightly along the fiber so that the moved $s_{1}(B L)$ intersects $s_{2} h(D)$ transversely in a small neighborhood of $\left(x_{i}, x_{i}, L_{x_{i}}, x_{i}\right)$ and count the intersection points in this neighborhood. Since this is a local problem, we can analyze it as follows. Take an $\varepsilon$-neighborhood $B L_{\varepsilon}$ of $\left(x_{i}, x_{i}, L_{x_{i}}\right)$ in $B L$ and an $\varepsilon$-neighborhood $\tilde{D}_{\varepsilon}$ of $\left(x_{i}, x_{i}, L_{x_{i}}\right)$ in $\tilde{D}$. Then move $s_{1}\left(B L_{\varepsilon}\right)$ slightly along the fiber and count the intersection points of the moved $s_{1}\left(B L_{\varepsilon}\right)$ and $s_{2} h\left(\tilde{D}_{\varepsilon}\right)$. To be more precise, let $H$ be a hyperplane not containing $x_{i}$ and consider the affine variety $X^{a}=X-H \subset \mathbf{P}^{N}-H=\mathbf{C}^{N}$. Here we can assume that a generic point $P$ does not lie on $H$, so $P \in \mathbf{C}^{N}$. Let $\pi: B L \rightarrow \mathbf{C}^{N} \times \mathbf{C}^{N}$ be the blowup (we use the same symbol $B L$ which denotes $\left.\mathbf{P}^{N} \times \mathbf{P}^{N}\right)$ and let $\left(\mathbf{a}^{0}, \mathbf{a}^{0}, \boldsymbol{\theta}^{0}\right)$ correspond to the point $\left(x_{i}, x_{i}, L_{x_{i}}\right)$. So $x_{i}$ corresponds to the point $\mathbf{a}^{0}$ under the isomorphism $\mathbf{P}^{N}-H \cong \mathbf{C}^{N}$. Without loss of generality, we can assume that the first homogeneous coordinate $\theta_{1}^{0}$ is not zero and consider the affine coordinates $\eta_{i}=\theta_{i} / \theta_{1}$ $(2 \leqslant i \leqslant N)$. Then, let $\left(\mathbf{a}^{0}, \mathbf{a}^{0}, \boldsymbol{\eta}^{0}\right)$ be corresponding to $\left(\mathbf{a}^{0}, \mathbf{a}^{0}, \boldsymbol{\theta}^{0}\right)$. Let

$$
\begin{aligned}
& B L_{\boldsymbol{\varepsilon}}=\left\{(\mathbf{z}, \mathbf{w}, \boldsymbol{\eta}) \mid z_{i}-w_{i}=\left(z_{1}-w_{1}\right) \cdot \eta_{i},\right. \\
&\left.\left\|\mathbf{z}-\mathbf{a}^{0}\right\|<\varepsilon,\left\|\mathbf{w}-\mathbf{a}^{0}\right\|<\varepsilon,\left\|\boldsymbol{\eta}-\boldsymbol{\eta}^{0}\right\|<\varepsilon\right\}
\end{aligned}
$$

where

$$
\left\|\mathbf{z}-\mathbf{a}^{0}\right\|=\sqrt{\left|z_{1}-a_{1}^{0}\right|^{2}+\cdots+\left|z_{N}-a_{N}^{0}\right|^{2}}
$$

is the distance between $\mathbf{z}$ and $\mathbf{a}^{0}$, and so for $\left\|\mathbf{w}-\mathbf{a}^{0}\right\|$ and $\left\|\boldsymbol{\eta}-\eta^{0}\right\|$. Let $\tilde{D}_{\varepsilon}=\tilde{D} \cap$ $B L_{\varepsilon}$. Let $t$ be a sufficiently small complex number such that $0<\|t\| \ll \varepsilon$. Then let

$$
s_{1, t}\left(B L_{\varepsilon}\right)=\text { closure of }\left\{\left(\mathbf{z}, \mathbf{w}, \boldsymbol{\eta}, \mathbf{z}+t \cdot \frac{\mathbf{w}-\mathbf{z}}{\|\mathbf{w}-\mathbf{z}\|}\right) \mid \mathbf{z} \neq \mathbf{w},(\mathbf{z}, \mathbf{w}, \boldsymbol{\eta}) \in B L_{\varepsilon}\right\}
$$

where $\mathbf{z}+t \cdot(\mathbf{w}-\mathbf{z}) /\|\mathbf{w}-\mathbf{z}\|$ means

$$
\left(z_{1}+t \cdot \frac{w_{1}-z_{1}}{\|\mathbf{w}-\mathbf{z}\|}, \ldots, z_{N}+t \cdot \frac{w_{N}-z_{N}}{\|\mathbf{w}-\mathbf{z}\|}\right) .
$$

Here the fourth entry $\mathbf{z}+t \cdot(\mathbf{w}-\mathbf{z}) /\|\mathbf{w}-\mathbf{z}\|$ does make sense because we can consider $s_{1, t}\left(B L_{\varepsilon}\right)$ in the affine trivial $C^{N}$-bundle over $B L$ (cf. the bundle $K$ described before). For the moment we assume that $s_{1, t}\left(B L_{\varepsilon}\right)$ is transverse to $s_{2} h\left(\tilde{D}_{\varepsilon}\right)$, which will be discussed later. Then the number of intersection points of 
$s_{2} h\left(\tilde{D}_{\varepsilon}\right) \cap s_{1, t}\left(B L_{\varepsilon}\right)$ is given by

$$
\begin{aligned}
& \#\left\{(\mathbf{z}, \mathbf{w}, \boldsymbol{\eta}, w) \in s_{2} h\left(\tilde{D}_{\varepsilon}\right) \mid \mathbf{w}=\mathbf{z}+t \cdot \frac{\mathbf{w}-\mathbf{z}}{\|\mathbf{w}-\mathbf{z}\|}\right\} \\
& =\#\left\{(\mathbf{z}, \mathbf{w}, \boldsymbol{\eta}) \in \tilde{D}_{\varepsilon}|\|\mathbf{w}-\mathbf{z}\|=| t \mid\right\} \\
& =\#\left\{\mathbf{w} \in X^{a} \cap B_{\varepsilon}\left(\mathbf{a}^{0}\right)|| t \mid=\|\mathbf{z}-\mathbf{w}\|, \exists \mathbf{z} \in X^{a} \cap B_{\varepsilon}\left(\mathbf{a}^{0}\right), \widehat{\mathbf{z w}} \ni P\right\},
\end{aligned}
$$

where $\widehat{\mathbf{z w}}$ is the line going through $\mathbf{z}$ and $\mathbf{w}$.

Here we notice that the transversality of $s_{2} h\left(\tilde{D}_{\varepsilon}\right)$ and $s_{1, t}\left(B L_{\varepsilon}\right)$ implies the transversality of $s_{P, t}\left(X^{a}\right)$ and $X^{a}$ within $B_{\varepsilon}\left(\mathbf{a}^{0}\right)$. Hence the following does make sense:

$$
\begin{aligned}
\#\left(S_{P, t}\left(X^{a}\right) \cap X^{a} \cap B_{\varepsilon}\left(\mathbf{a}^{0}\right)\right)=\#\left\{\mathbf{w} \in X^{a} \cap B_{\varepsilon}\left(\mathbf{a}^{0}\right) \mid \mathbf{w}=\mathbf{z}+t \cdot \frac{P-\mathbf{z}}{\|P-\mathbf{z}\|}\right\} \\
=\#\left\{\mathbf{w} \in X^{a} \cap B_{\varepsilon}\left(\mathbf{a}^{0}\right)|| t \mid=\|\mathbf{w}-\mathbf{z}\|, \exists \mathbf{z} \in X^{a} \cap B_{\varepsilon}\left(\mathbf{a}^{0}\right), \widehat{\mathbf{z w}} \ni P\right\} .
\end{aligned}
$$

Thus

$$
n_{i}=\#\left(S_{P, t}\left(X^{a}\right) \cap X^{a} \cap B_{\varepsilon}\left(\mathbf{a}^{0}\right)\right)=n\left(x_{i}, P, t, \varepsilon\right) .
$$

Therefore by Observation 5.8, (5.12), and since taking a generic center $P$ leads us to formula (5.3') (although it is independent of the choice of a generic center $P$ ), we can conclude both statements (i) and (ii) of Theorem 5.6. This completes the proof of the theorem.

It remains only to prove

LEMMA 5.11. $s_{1, t}\left(B L_{\varepsilon}\right)$ is transverse to $s_{2} h\left(\tilde{D}_{\varepsilon}\right)$ for a sufficiently small $\varepsilon$.

Proof. (Cf. Figure 5.) Let $\Gamma$ be a sufficiently small branch of $s_{1} h\left(\tilde{D}_{\varepsilon}\right)$ at the point $s_{1}(a)=s_{2}(a)$, so $\Gamma-\left\{s_{1}(a)\right\}$ is smooth. For a sufficiently small $\varepsilon$, we may consider that $s_{1, t}\left(B L_{\varepsilon}\right)$ and $s_{2} h\left(\tilde{D}_{\varepsilon}\right)$ are in the product $\mathbf{C}^{2 N} \times \mathbf{C}$, where the second factor $\mathbf{C}$

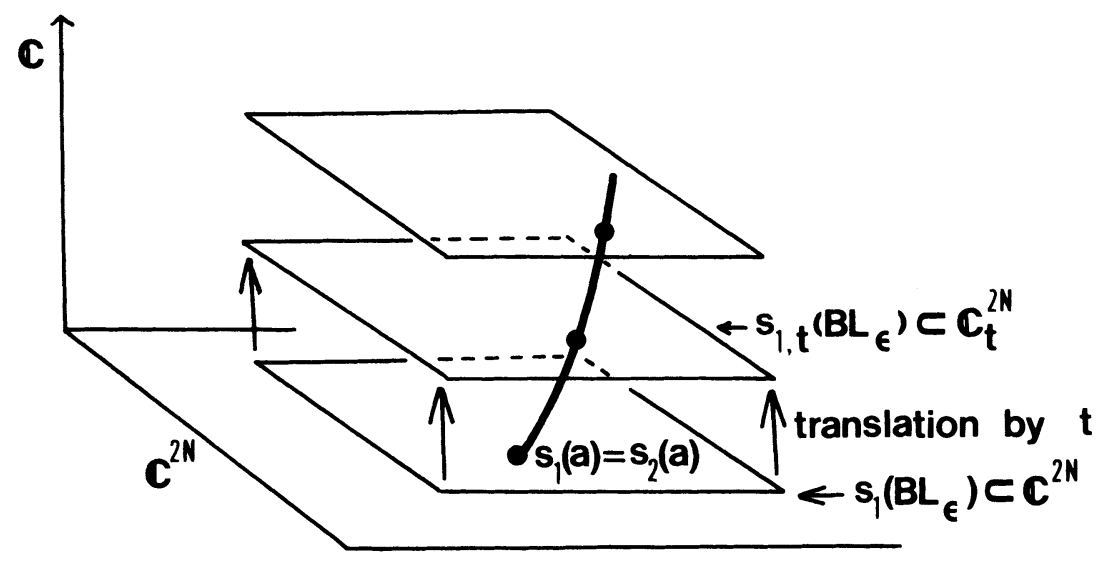

Figure 5 
indicates the fiber, and $s_{1}\left(B L_{\varepsilon}\right)$ are in the first factor $C^{2 N}$, and $s_{1, t}\left(B L_{\varepsilon}\right)$ is the translation of $s_{1}\left(B L_{\varepsilon}\right)$ along the fiber $\mathbf{C}$ by $t$. Let $\Delta$ be a small closed disk around the origin in the complex line $\mathbf{C}_{w}$ where $w$ denotes the coordinate of $\mathbf{C}$, and let $f$ : $(\Delta, 0) \rightarrow\left(\Gamma, s_{1}(a)\right)$ be the local uniformization (or parameterization) of $\Gamma$; i.e., $\left(f(w)=\left(f_{1}(w), \ldots, f_{2 N}(w), f_{2 N+1}(w)\right)\right.$, where $f_{i}(w)$ is a holomorphic function. Let $\pi: \mathbf{C}^{2 N} \times \mathbf{C} \rightarrow \mathbf{C}$ be the projection map, which is clearly holomorphic. Then under the local uniformization of $\Gamma$, the points where $\Gamma$ are not transverse to $s_{1, t}\left(B L_{\varepsilon}\right)$, i.e., the points where the tangent lines of $\Gamma$ are in the translated $\mathbf{C}_{t}^{2 N}$ of $\mathbf{C}^{2 N}$, correspond to the points $w$ of $\Delta$ such that $(\pi \circ f)^{\prime}(w)=0$. Since $\pi \circ f: \Delta \rightarrow \mathbf{C}$ is holomorphic and not a constant map, there are not infinitely many such points $w \in \Delta$ as $(\pi \circ f)^{\prime}(w)=0$; otherwise, by a well-known fact in analytic function theory (i.e., identity theorem), $(\pi \circ f)^{\prime} \equiv 0$, which implies that $\pi \circ f$ is constant, a contradiction. Thus, for each branch (there are only finitely many branches of $s_{2} h\left(\tilde{D}_{\varepsilon}\right)$ at the point $\left.s_{1}(a)=s_{2}(a)\right)$, there are only finitely many points where the branch is not transverse to $s_{1, t}\left(B L_{\varepsilon}\right)$. Hence if we take a sufficiently small $\varepsilon$ and $t, s_{1, t}\left(B L_{\varepsilon}\right)$ is always transverse to $s_{2} h\left(\tilde{D}_{\varepsilon}\right)$. This completes the proof of the lemma.

\section{BIBLIOGRAPHY}

[Du] A. Dubson, Calcul des invariants numeriques des singularités et applications, S.T.B. Theor. Math., Universität Bonn, 1981.

[E] M. Eger, Sur les systèmes canoniques d'une variété algébrique, C. R. Acad. Sci. Paris. Sér. I Math. 204 (1937), 217-219.

[F1] W. Fulton, Rational equivalence on singular varieties, Inst. Hautes Études Sci. Publ. Math. 45 (1975), 147-167.

[F2] , On the fundamental group of the complement of a node curve, Ann. of Math. (2) 111 (1980), 407-409.

[F3] Introduction to intersection theory in algebraic geometry, Lecture notes prepared for NSF-CBMS Regional Conf. on Intersection Theory in Algebraic Geometry, George Mason Univ., June 27-July 1, 1983.

[F4] Intersection theory, Ergebnisse Math., 3 Folge, vol. 2, Springer-Verlag, Berlin, 1984.

[FL] W. Fulton and D. Laksov, Residual intersections and the double point formula, Real and Complex Singularities (Oslo, 1976), Sijthoff and Noordhoff, 1977, pp. 171-177.

[FM1] W. Fulton and R. MacPherson, Intersection cycles on an algebraic variety, Real and Complex Singularities (Oslo, 1976), Sijthoff and Noordhoff, 1977, pp. 179-197.

[FM2] _ Defining algebraic intersections, Algebraic Geometry (Tromsø, Norway, 1977), Lecture Notes in Math., vol. 687, Springer-Verlag, Berlin and New York, 1978, pp. 1-30.

[GH] P. Griffiths and J. Harris, Principles of algebraic geometry, Wiley, New York, 1978.

[Go] G. Gonzalez-Sprinberg, L'obstruction locale d'Euler et le théorème de MacPherson, Séminaire E.N.S., Exposé no. 1, 1978-1979, pp. 7-32.

[Ho1] A. Holme, Embedding obstruction for smooth, projective varieties. I, Studies in Algebraic Topology (G.-C. Rota, ed.), Adv. in Math. Suppl. Stud., Vol. 5, Academic Press, New York, 1979, pp. 39-67.

[Ho2] $155-185$ Embedding obstruction for singular algebraic varieties in $\mathbf{P}^{N}$, Acta. Math. 135 (1976),

[HR] A. Holme and Joel Roberts, Pinch-points and multiple locus of generic projections of singular varieties, Adv. in Math. 33 (1979), 212-256.

[Jo] K. Johnson, Immersion and embedding of projective varieties, Acta Math. 140 (1978), 49-74.

[K1] S. L. Kleiman, The enumerative theory of singularities, Real and Complex Singularities (Oslo, 1976), Sijthoff and Noordhoff, 1977, pp. 297-396.

[KL] S. L. Kleiman and D. Laksov, Schubert calculus, Amer. Math. Monthly 79 (1972), 1061-1082.

[LT] Lê Dũng Tràng and $\mathrm{B}$. Teissier, Variètés polaire locales et classes de Chern des variétés singulières, Ann. of Math. (2) 114 (1981), 457-491. 
[Mac] R. MacPherson, Chern classes for singular varieties, Ann. of Math. (2) 100 (1974), 423-432.

[Pil] R. Piene, Polar classes of singular varieties, Ann. Sci. École Norm. Sup. (4) 11 (1978), 247-276.

[Pi2] __. Cycles polaires et classes de Chern, Séminaire du les singularités des surfaces, 1977-1978, Ecole Polytéchnique, Palaiseau, France, pp. 1-26.

[Se] J. P. Serre, Algèbre locale-multiplicités, Lecture Notes in Math., vol. 11, Springer-Verlag, Berlin and New York, 1965.

[Sh] T. Shifrin, The kinematic formula in complex integral geometry, Trans. Amer. Math. Soc. 264 (1981), 255-293.

[To1] J. A. Todd, The arithmetical invariants of algebraic loci, Proc. London Math. Soc. 43 (1937), $190-225$.

[To2] _ Invariant and covariant systems on an algebraic variety, Proc. London Math. Soc. 46 (1940), 199-230.

[U1] T. Urabe, Duality of numerical characters of polar loci, Publ. Res. Inst. Math. Sci. Kyoto Univ. 17 (1981), 331-345.

[U2] Generalized Plücker formulae, Publ. Res. Inst. Math. Sci. Kyoto Univ. 17 (1981), 347-362.

[Wh] H. Whitney, Complex analytic varieties, Addison-Wesley, Reading, Mass., 1972.

Department of Mathematics, University of Georgia, Athens, Georgia 30602

Current address: Faculty of Engineering, University of Kagoshima, 1-21-40 Korimoto, Kagoshima 890, Japan 